\title{
PKC $\alpha$ regulates vasopressin-induced aquaporin-2 trafficking in mouse kidney collecting duct cells in vitro via altering microtubule assembly
}

\author{
Hong ZHAO ${ }^{1, \#}$, Xi YAO ${ }^{2, \#}$, Tao-xia WANG ${ }^{2}$, Wen-min JIN ${ }^{1}$, Qian-qian $J^{1}$, Xiao YANG ${ }^{1}$, Qiu-hong DUAN ${ }^{3}$, Li-jun YAO ${ }^{2, *}$ \\ ${ }^{1}$ Department of Trauma Surgery, Tongji Hospital, Huazhong University of Science \& Technology, Wuhan 430030, China; ${ }^{2}$ Departments \\ of Nephrology, Union Hospital, Huazhong University of Science \& Technology, Wuhan 430030, China; ${ }^{3}$ Department of Biochemistry \\ and Molecular Biology, Tongji Medical College, Huazhong University of Science \& Technology, Wuhan 430022, China
}

Aim: Aquaporin-2 (AQP2) is a vasopressin-regulated water channel located in the collecting tubule and collecting duct cells of mammalian kidney. The aim of this study is to investigate whether PKC $\alpha$ plays a role in vasopressin-induced AQP2 trafficking in mouse inner medullary collecting duct 3 (mIMCD3) cells.

Methods: AQP2-mIMCD3 stable cell line was constructed by transfection of mouse inner medullary collecting duct 3 (mIMCD3) cells with AQP2-GFP construct. Then the cells were transfected with PKC $\alpha$ shRNA, PKC $\alpha$ A/25E, or PKC $\alpha$ scrambled shRNA. The expression levels of PKC $\alpha, A Q P 2$, and phospho-S256-AQP2 were analyzed using Western blot. The interaction between AQP2 and PKC $\alpha$ was examined using immunoprecipitation. The distribution of AQP2 and microtubules was studied using immunocytochemistry. The AQP2 trafficking was examined using the biotinylation of surface membranes.

Results: Treatment of AQP2-mIMCD3 cells with $100 \mu \mathrm{mol} / \mathrm{L}$ of 1-desamino-8-D-arginine vasopressin (DdAVP) for 30 min stimulated the translocation of AQP2 from the cytoplasm to plasma membrane through influencing the microtubule assembly. Upregulation of active PKC $\alpha$ by transfection with PKC $\alpha$ A/25E plasmids resulted in de-polymerization of $\alpha$-tubulin and redistributed AQP2 in the cytoplasm. Down-regulation of PKC $\alpha$ by PKC $\alpha$ shRNA partially inhibited DdAVP-stimulated AQP2 trafficking without altering $\alpha$-tubulin distribution. Although $100 \mu \mathrm{mol} / \mathrm{L}$ of DdAVP increased AQP2 phosphorylation at serine 256, down-regulation of PKC $\alpha$ by PKC $\alpha$ shRNA did not influence DdAVP-induced AQP2 phosphorylation, suggesting that AQP2 phosphorylation at serine 256 was independent of PKC $\alpha$. Moreover, PKC $\alpha$ did not physically interact with AQP2 in the presence or absence of DdAVP.

Conclusion: Our results suggested that PKC $\alpha$ regulates AQP2 trafficking induced by DdAVP via microtubule assembly.

Keywords: PKC $\alpha$; 1-desamino-8-D-arginine vasopressin (DdAVP); aquaporin-2; microtubule; kidney; medullary collecting duct

Acta Pharmacologica Sinica (2012) 33: 230-236; doi: 10.1038/aps.2011.160; published online 2 Jan 2012

\section{Introduction}

Protein kinase $\mathrm{C}$ alpha (PKCa) is a member of the classic PKC family ${ }^{[1]}$, which is widely expressed in the mammalian kidney ${ }^{[2-4]}$ and contributes to various kidney functions, including substrate absorption and urine concentration ${ }^{[5,6]}$. PKCamediated cytoskeleton remodeling results in endocytosis in epithelial cells, which increases the uptake of various substrates, such as NHE3 and albumin ${ }^{[7-9]}$. PKCa is localized in glomeruli, the intercalated cells of the cortical collecting duct, and the medullary-collecting duct in the mouse kidney ${ }^{[4]}$. Genetic knockout mice that lack PKCa exhibit decreased

\footnotetext{
\# These two authors contributed equally to this paper.

* To whom correspondence should be addressed.

E-mail drylj@hotmail.com

Received 2011-06-28 Accepted 2011-10-28
}

glomerular filtration rate, increased urinary output and lower urinary osmolarity, accompanied by normal water intake and normal levels of the plasma antidiuretic hormone arginine vasopressin $(\mathrm{AVP})^{[5]}$. These results strongly suggest that PKCa-mediated urine concentration primarily occurs within the medullary collecting duct.

Aquaporin-2 (AQP2) is a vasopressin-regulated water channel in the principal cells of the connecting tubule and the collecting duct in the kidney ${ }^{[10,11]}$. AQP2 is stored in an intracellular compartment and plays an important role in the regulation of urine concentration ${ }^{[12-15]}$. AVP stimulates AQP2 translocation to the plasma membrane for the re-absorption of water. AQP2 is removed from the plasma membrane and returned to the intracellular compartment when the stimulation in terminated ${ }^{[16-18]}$. In addition, AVP also regulates AQP2 mRNA and protein levels, which mediates long-term regula- 
tion $^{[19,20]}$. Both short- and long-term regulation mechanisms are involved in the pathophysiology of AQP2-mediated urine concentration $^{[21]}$. Although PKC is involved in angiotensin II-mediated AQP2 expression and trafficking ${ }^{[22]}$, our previous study demonstrated that AQP2 expression is only slightly different between PKCa knockout mice and wild-type mice ${ }^{[5]}$, which strongly suggests that the long-term AQP2 regulation mechanism does not contribute to PKCa-mediated urine concentration. AQP2 transportation is partially regulated by the cytoskeleton ${ }^{[23-25]}$, and PKCa-mediated remodeling of the actin cytoskeleton is involved in constitutive albumin uptake in the renal proximal tubule ${ }^{[9]}$. However, the contribution of PKCa to AQP2 trafficking, the role of the cytoskeleton in PKCamediated AQP2 transportation and the mechanism of PKCa regulation of AQP2 translocation are less well understood.

We hypothesized that PKCa mediates AQP2 trafficking via cytoskeleton distribution because of the important role of PKCa in urine concentration and cytoskeleton remodeling. Therefore, this study revealed the crosstalk among PKCa, AVP and AQP2 in trafficking and cytoskeletal remodeling in mIMCD3 cell.

\section{Materials and methods Constructs, antibodies, and reagents}

The pEF-nero-PKCa A/25E vector was a gift from Dr Gottfried BAIER (Institute for Medical Biology and Human Genetics, University of Innsbruck, Innsbruck, Austria). The AQP2GFP-pCMV6 construct was purchased from Origene (Rockville, MD, USA). Lipofectamine plus and geneticin (G418) were purchased from Invitrogen (Shanghai, China). Protein A/G-agarose beads and primary antibodies against phosphorS256-AQP2, AQP2, PKCa, and GAPDH were purchased from Santa Cruz (Heidelberg, Germany). The primary antibody against a-tubulin was purchased from Calbiochem (Darmstadt, Germany). The secondary goat anti-rabbit IgG conjugated with Cy3 antibody and the analogue 1-desamino-8-Darginine vasopressin (DdAVP) were purchased from Sigma (Shanghai, China). DAPI was purchased from Vector Burlingame (San Diego, CA, USA). Sulfo-Link NHS-LC-biotin and streptavidin-agarose beads were purchased from Pierce (Beijing, China). Major apparatuses included a Mini-PROTEAN II Electrophoresis Cell (Bio-Rad, Shanghai, China) and a confocal laser-scanning microscope (Olympus FV500, Japan).

\section{Cells and cell culture}

Immortalized mouse inner medullary collecting duct 3 (mIMCD3) cells were kindly provided by Dr John M LUK (Department of Urology, University of Hong Kong, Hong Kong, China). mIMCD3 cells were maintained in Dulbecco's modified Eagle's medium/Ham's F12 (1:1) (GIBCO, Invitrogen, Shanghai, China) supplemented with $10 \%$ fetal bovine serum (GIBCO, Invitrogen, Shanghai, China) and 2\% penicillin-streptomycin (Amresco, Shanghai, China) in a humidified atmosphere with $5 \% \mathrm{CO}_{2}$ at $37^{\circ} \mathrm{C}$.

\section{Generation of pGCsi-U6/Neo PKC $\alpha$ shRNA plasmid}

Three siRNAs were designed and synthesized by Invitrogen (Shanghai, China) according to the cDNA sequence of PKCa (NM_011101): \#1, GTCCTTCACGTTCAAATTA; \#2, GTGCAGTATGAAACTCAAA; and \#3, CCATCCAACAACCTGGACA. The siRNAs were cloned into the eukaryotic expression plasmid pGCsi-U6/Neo (Genechem, Shanghai, China). The constructed pGCsi-U6/Neo-PKCa-siRNA vector was transfected into mIMCD3 cells. Western blotting was used to evaluate the suppression of PKCa expression in different cell groups.

\section{Transfection}

Transfection of mIMCD3 cells with the AQP2-GFP construct was performed using Lipofectamine 2000 (Shanghai, China) according to the manufacturer's instructions. The cells were maintained in medium containing $400 \mu \mathrm{g} / \mathrm{mL}$ geneticin for $24 \mathrm{~h}$ after transfection. Individual neomycin-resistant colonies were selected and expanded for $14 \mathrm{~d}$ after transfection.

AQP2-GFP was stably expressed in mIMCD3 cells (AQP2mIMCD3 cells) that were transiently transfected with a eukaryotic expression vector encoding the constitutively activated form of PKCa A/25E (pEF-nero-PKCa A/25E) ${ }^{[26]}$ using Lipofectamine 2000 according to the manufacturer's instructions. AQP2-mIMCD3 cells were transfected with three pGCsi-U6/Neo-PKCa shRNAs and scrambled shRNA using Lipofectamine 2000 according to the manufacturer's instructions to evaluate the inhibition efficiency of the siRNAs. The cells were harvested $48 \mathrm{~h}$ after transfection, and Western blot and immunofluorescence were used to evaluate the transfection efficiency.

\section{Immunocytochemical staining}

AQP2-mIMCD3 cells were divided into 3 groups: (1) Scrambled shRNA transfected; (2) PKCa A/25E vector transfected; and (3) PKCa shRNA transfected. The cells in each group were treated with $100 \mu \mathrm{mol} / \mathrm{L}$ DdAVP for $30 \mathrm{~min}$. DdAVP was removed, and the cells were incubated with culture medium for $2 \mathrm{~h}$ (washout). The cells were then fixed in $4 \%$ paraformaldehyde for $10 \mathrm{~min}$, rinsed twice in PBS, and blocked for $15 \mathrm{~min}$ in a blocking/permeabilization solution (PBS containing $0.1 \%$ BSA and $0.3 \%$ Triton X-100). The cells were washed with PBS and pre-incubated in PBS containing $1 \%$ BSA for $20 \mathrm{~min}$. The cells were incubated with an a-tubulin antibody (at a final concentration of $2 \mu \mathrm{g} / \mathrm{mL}$ ) at $4{ }^{\circ} \mathrm{C}$ overnight. The cells were washed with PBS and incubated in the secondary goat anti-rabbit IgG conjugated with Cy3 antibody (at a final concentration of $2 \mu \mathrm{g} / \mathrm{mL}$ ) for $1.5 \mathrm{~h}$ at RT. Finally, the cells were washed twice with PBS and mounted with DAPI for confocal laser-scanning microscopy equipped with a CoolSNAP HQ camera.

\section{Biotinylation of surface membrane proteins}

Identifiably transfected AQP2-mIMCD3 cells were cultured on polylysine-coated $60-\mathrm{mm}$ dishes for $48 \mathrm{~h}$ and treated with 
DdAVP for $0 \mathrm{~min}, 30 \mathrm{~min}$, or $30 \mathrm{~min}$ followed by a 2-h washout. The cells were washed three times with PBS/glycine (137 $\mathrm{mmol} / \mathrm{L} \mathrm{NaCl}, 2.7 \mathrm{mmol} / \mathrm{L} \mathrm{KCl}, 10 \mathrm{mmol} / \mathrm{L} \mathrm{Na}_{2} \mathrm{HPO}_{4}, 1.8$ $\mathrm{mmol} / \mathrm{L} \mathrm{KH}_{2} \mathrm{PO}_{4}, \mathrm{pH} 8.0$, and $5 \mathrm{mmol} / \mathrm{L}$ glycine) and twice with $\mathrm{PBS}^{[27]}$. An ice-cold biotinylation reagent $(0.5 \mathrm{mg} / \mathrm{mL}$ in PBS) was immediately added to cells. The biotinylation reagent was removed after a 10 -min incubation on ice, and the cells were washed three times with PBS/glycine.

\section{Immunoblotting and immunoprecipitation}

Transfected and DdAVP-treated cells were lysed in RIPA buffer $(10 \mathrm{mmol} / \mathrm{L}$ Tris-HCl, $0.15 \mathrm{~mol} / \mathrm{L} \mathrm{NaCl}, 1 \% \mathrm{NP}-40,1 \% \mathrm{Na}-$ deoxycholate, $0.5 \%$ SDS, $0.02 \%$ sodium azide, and $1 \mathrm{mmol} / \mathrm{L}$ EDTA, pH 7.4). The cell lysates were clarified by centrifugation at $1000 \times g$ for $10 \mathrm{~min}$ at $4^{\circ} \mathrm{C}$. Protein concentrations were measured using the bicinchoninic acid protein assay reagent kit (Pierce, Thermo Fisher Scientific, Beijing, China) and adjusted to the same concentration using lysine buffer. A $0.5-\mathrm{mL}$ aliquot of the cell lysates was incubated with $50 \mu \mathrm{L}$ of streptavidin-agarose beads at $4{ }^{\circ} \mathrm{C}$ overnight to capture biotinylated proteins, $50 \mu \mathrm{L}$ of protein $\mathrm{A} / \mathrm{G}$-agarose beads plus the AQP2 antibody to capture total AQP2, or $50 \mu \mathrm{L}$ of protein A/G-agarose beads plus the PKCa antibody to capture total PKCa. The beads were pelleted, washed, and boiled for $5 \mathrm{~min}$ in $50 \mu \mathrm{L}$ of cracking buffer $(50 \mathrm{mmol} / \mathrm{L}$ Tris- $\mathrm{HCl}$ [pH 7.0], $10 \%$ glycerol, $2 \%$ SDS, and $2 \% \beta$-mercaptoethanol). The eluted immunoprecipitates were separated on $10 \%$ polyacrylamide gels and transferred electrophoretically to PVDF membrane. The membranes were blocked for 90 min with blocking buffer during gentle shaking and incubated overnight with antibodies against AQP2 $(0.5 \mu \mathrm{g} / \mathrm{mL})$, phospho-S256-AQP2 (0.5 $\mu \mathrm{g} / \mathrm{mL})$, PKCa $(0.5 \mu \mathrm{g} / \mathrm{mL})$ or GAPDH $(0.5 \mu \mathrm{g} / \mathrm{mL})$. The membranes were incubated with an HRP-conjugated IgG (0.1 $\mu \mathrm{g} / \mathrm{mL}$ ) for $2 \mathrm{~h}$ at room temperature. Finally, the immunoreactive signals were visualized using an ECL reagent (Beyotime, Shanghai, China) and quantified using the Image J program (NIH, Bethesda, MD, USA).

\section{Statistical analyses}

The values are presented as the means \pm SEM. The data were analyzed using SPSS software. Statistical significance of differences was assessed using unpaired $t$-tests or two-way ANOVAs. A value of $P<0.05$ was viewed as statistically significant.

\section{Results}

Inhibition of PKC $\alpha$ and the overexpression of AQP2 in mIMCD3 cells

All three PKCa shRNA plasmids (\#1, \#2, and \#3) and PKCa scrambled shRNA plasmids were transfected into mIMCD3 cells to select the shRNAs with the highest inhibition efficiency to PKCa. Crude proteins were extracted from the harvested cells $48 \mathrm{~h}$ after transfection and subjected to Western blotting using the PKCa antibody. PKCa shRNA \#1 plasmids exhibited the greatest inhibitory effect on PKCa, but all three PKCa shRNAs demonstrated visible inhibition $(P<0.01)$ (Figure $1 \mathrm{~A})$.

The transfection of mIMCD3 with the AQP2-GFP-pCMV6 plasmid produced 12 geneticin-resistant clones, 10 of which were subjected to Western blot analysis (data not shown). The clone that amply expressed AQP2 was selected and named WT-7. A strong signal at $30 \mathrm{kDa}$ in the selected AQP2mIMCD3 stable cell line (WT-7) was observed, but no signal was detected in nontransfected mIMCD3 cells (Figure 1B).
A
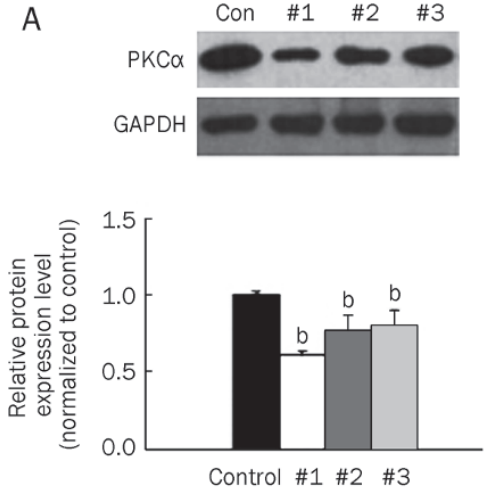

$B$

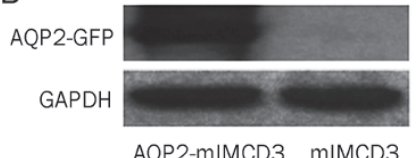

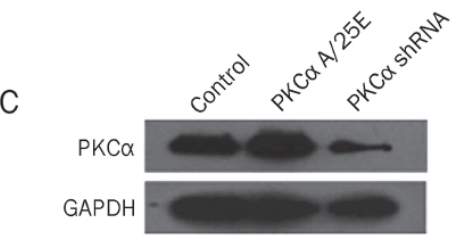

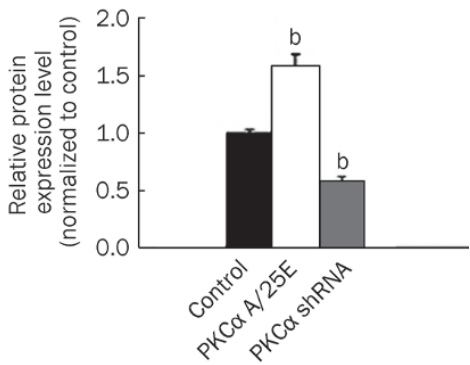

Figure 1. Inhibition of PKC $\alpha$ and the overexpression of AQP2 in mIMCD3 cells. (A) PKC $\alpha$ shRNA plasmid \#1, \#2, \#3, and PKC $\alpha$ scramble shRNA plasmid (control) were transfected into mIMCD3 cells respectively. Forty-eight hours after transfection, crude proteins extracted from harvested cells were subjected to Western blot using antibodies against PKC $\alpha$. Representative blot was shown (left) and quantitative analysis of PKC $\alpha$ protein levels were normalized to control group ( $n=3$ each group) (right). ${ }^{b} P<0.05$ vs control group. (B) AQP2-mIMCD3 stable cell line was generated by transfecting to express AQP2-GFP (See Materials and methods). The expression of AQP2-GFP was detected in selected WT7 stable cell lines. (C) AQP2-GFP stably expressed WT7 cells were transfected with scramble shRNA (Con), PKC $\alpha$ A/25E constructs or PKC $\alpha$ shRNA, respectively. Forty-eight hours after transfection, crude proteins extracted from harvested cells were subjected to Western blot using antibodies against PKC $\alpha$ (left). Quantitative analysis of $\mathrm{PKC} \alpha$ protein levels were normalized to control group ( $n=3$ each group) (right). ${ }^{\mathrm{b}} P<0.05$ vs control group. 
These results suggest the successful preparation of AQP2mIMCD3 stable cell lines.

The WT-7 stable cell line was used to investigate PKCa expression following different transfections of PKCa scrambled shRNA, PKCa A/25E, and PKCa shRNA (\#1) using Western blot. The transfection of PKCa A/25E significantly enhanced PKCa expression, but PKCa shRNA (\#1) dramatically suppressed PKCa expression $(P<0.05)$ (Figure $1 C)$. The WT-7 cell line and PKCa A/25E and PKCa shRNA (\#1) plasmids were utilized in subsequent experiments.

DdAVP enhanced the phosphorylation of AQP2 and PKC $\alpha$ in vitro The physical association of AQP2 with endogenous PKCa was examined in vitro in WT7 cell lines to reveal the direct role of PKCa on AQP2. Cells with or without DdAVP treatment for 30 min were lysed, and PKCa was immunoprecipitated using anti-PKCa antibodies. The immunoprecipitates were analyzed using Western blot and AQP2 antibodies. Strong signals for AQP2 and PKCa were detected in whole cell lysates and immunoprecipitates, but no AQP2 signal was detected in PKCa immunoprecipitates (Figure 2). These results suggest that PKCa did not interact with AQP2 in vitro. However, DdAVP treatment enhanced AQP2 phosphorylation at serine 256. Down-regulation of PKCa by shRNA did not alter DdAVP-mediated AQP2 phosphorylation at serine 256. These data indicated that PKCa did not influence DdAVP-induced AQP2 phosphorylation at serine 256 (Figure 2).

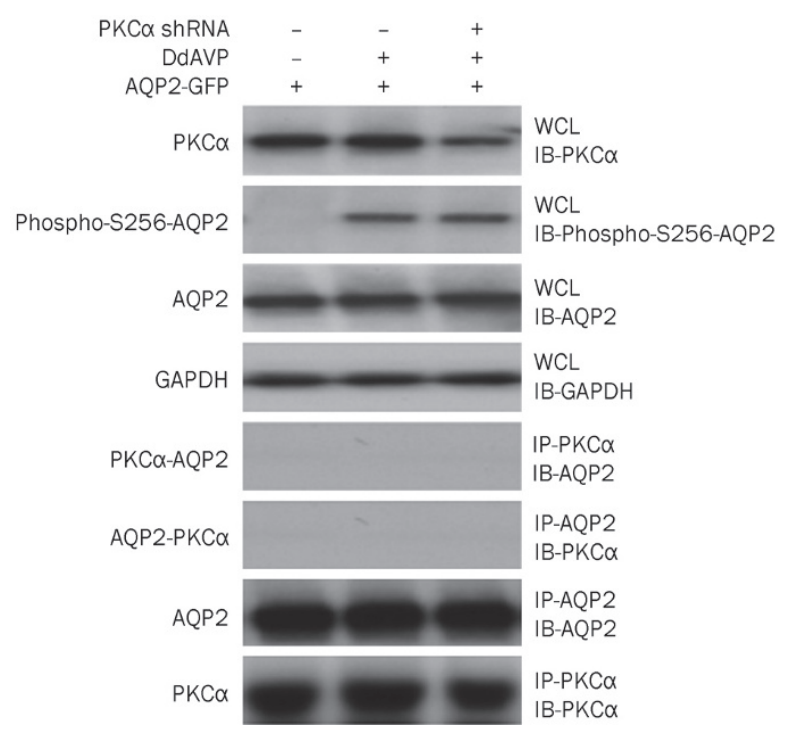

Figure 2. DdAVP enhanced the phosphorylation of AQP2 and AQP2 did not physically interact with PKC $\alpha$ in vitro. AQP2-GFP stably expressed WT7 cells were transfected with indicated constructs. Forty-eight hours after transfection, cells were treated with or without DdAVP for $30 \mathrm{~min}$. Cells were then lysed and immunoprecipitation was performed with anti-PKC $\alpha$ and anti-AQP2 antibodies. Crude proteins from whole cell lysates and the immunoprecipitates were subjected to Western blot with indicated antibodies.
PKC $\alpha$ expression influenced the distribution of $\alpha$-tubulin and DdAVP-mediated AQP2 trafficking in mIMCD cells

An increase in PKCa activity induces the re-organization of microtubules in proximal tubular cells ${ }^{[9]}$. Therefore, PKCainduced alterations in microtubule architecture were investigated. Most cells that were transfected with scrambled shRNA plasmid or PKCa shRNA plasmids exhibited a prominent dense network of microtubules around the nucleus with a considerably lower microtubule content in the cell periphery under basal conditions. The constitutive activation of PKCa increased microtubule formation in the cell periphery (Figure 3, upper panel and middle panel). AQP2-mIMCD3 cells (WT7 cells) were incubated with DdAVP for $30 \mathrm{~min}$ followed by a 2-h washout to evaluate the involvement of PKCa in AVP-induced microtubule formation in the cell periphery. Cells that were transfected with the control and PKCa A/25E plasmids displayed increased microtubule formation in the cell periphery following DdAVP stimulation. In contrast, the down-regulation of PKCa expression using shRNA inhibited DdAVP-induced microtubule depolymerization (Figure 3, lower panel). Taken together, these data strongly indicated that the expression of PKCa regulated microtubule distribution in mIMCD cells.

The role of PKCa in DdAVP-induced AQP2 trafficking was investigated because PKCa influences microtubule reorganization, and the DdAVP-induced depolymerization of microtubules affects the redistribution of AQP2 to the plasma membrane ${ }^{[25,28,29]}$. Immunofluorescence analyses indicated that AQP2 was primarily localized around the nucleus under basal condition. Treatment with DdAVP for $30 \mathrm{~min}$ produced a pronounced increase in AQP2 localization in the plasma membrane. DdAVP washout restored the perinuclear localization of AQP2. The expression of constitutively activated PKCa produced a wide distribution of AQP2 throughout the cytoplasm in contrast to AQP2 localization around the nucleus under basal conditions. DdAVP also induced the translocation of AQP2 to the plasma membrane in the PKCa A/25E transfected group. The down-regulation of PKCa expression by shRNA interference inhibited DdAVP-induced AQP2 translocation to the plasma membrane (Figure 3).

Regulation of PKC $\alpha$ expression influenced DdAVP-stimulated plasma location of AQP2

mIMCD-3 surface membrane proteins were biotinylated following DdAVP treatment for $0 \mathrm{~min}, 30 \mathrm{~min}$, and $30 \mathrm{~min}$ followed by a 2-h washout to confirm the immunofluorescence results. Biotinylated proteins were captured from whole-cell lysates using streptavidin beads, separated by SDS-PAGE and probed using an AQP2 antibody. Immunoprecipitation was performed in whole cell lysates in parallel using AQP2specific antibodies. The DdAVP-induced increase in biotinAQP2 was partially inhibited by PKCa down-regulation with shRNA transfection, but biotin-AQP2 was enhanced by the overexpression of PKCa A/25E (Figure 4). Moreover, different treatments only marginally affected the total amount of 

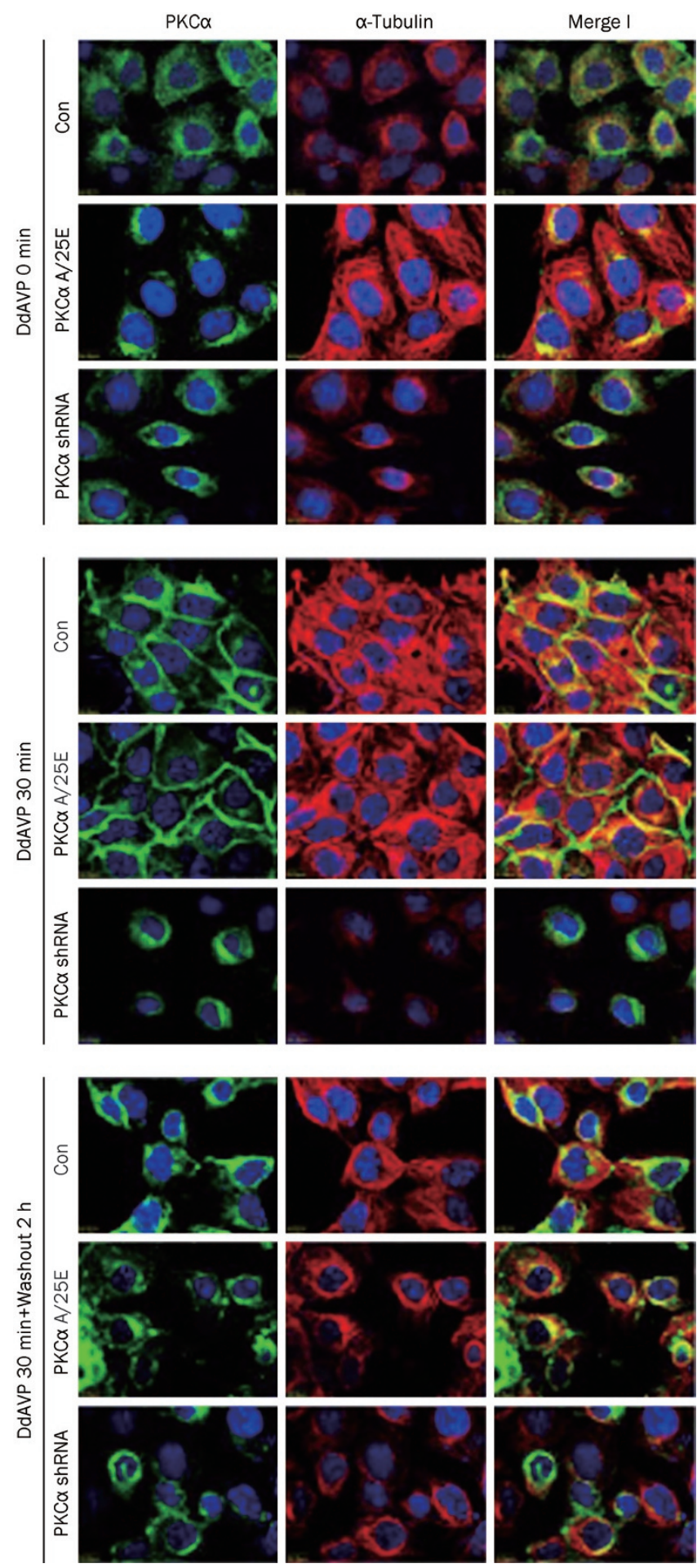

Figure 3. PKC $\alpha$ expression influenced the distribution of $\alpha$-tubulin and DdAVP-mediated AQP2 trafficking in mIMCD3 cells. AQP2-GFP stably expressed WT7 cells were transfected with PKC $\alpha$ cramble shRNA (Con), $P K C \alpha A / 25 E$ constructs or PKC $\alpha$ shRNA constructs. Forty-eight hours after transfection, cells were treated with or without DdAVP for $0 \mathrm{~min}, 30 \mathrm{~min}$ or $30 \mathrm{~min}$ followed by $2 \mathrm{~h}$ washout. Cells were then fixed, permeablized and stained with antibodies aganist PKC $\alpha$ (green) and $\alpha$-tubulin (red) for immunofluorescence. Nuclear was stained by DAPI (blue).
A
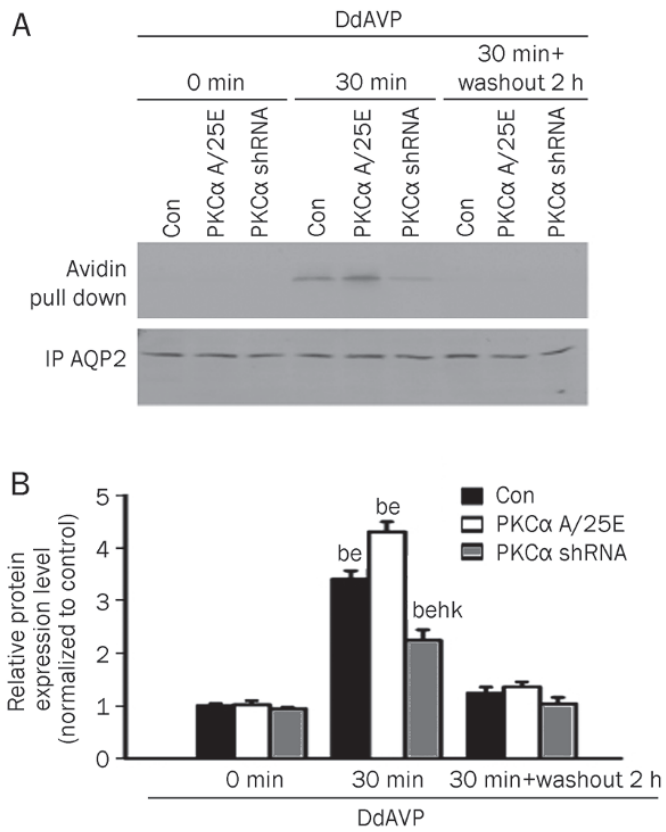

Figure 4. Regulation of $P K C \alpha$ expression influenced DdAVP stimulated plasma location of AQP2. (A) AQP2-GFP stably expressed WT7 cells were transfected with indicated constructs. Forty-eight hours after transfection, surface membrane proteins were biotinylated at $0 \mathrm{~min}$, DdAVP treated $30 \mathrm{~min}$, and DdAVP treated $30 \mathrm{~min}$ followed by $2 \mathrm{~h}$ washout. Whole cell lysates were subjected to avidin pulldown assay using streptavidinagarose beads or immunoprecipitation (IP) using anti-AQP2 antibodies. The recovered proteins were separated by SDS-PAGE and analyzed by Western blot for AQP2 as described in Materials and Methods. For each condition, the detected signals from avidin-pulldown assay and AQP2 immunoprecipitation were from same SDS-PAGE gel. (B) Signals from 3 independent experiments were quantified by densitometry. The intensity of the AQP2 signals from the avidin pulldowns were normalized to the corresponding AQP2 IP signals under each condition. Data were represented as mean \pm SEM. ${ }^{b} P<0.05$ vs DdAVP 0 min group. ${ }^{e} P<0.05$ vs DdAVP 30 min +2 h washout group. ${ }^{h} P<0.05$ vs DdAVP 30 min con group. ${ }^{k} P<0.05$ vs DdAVP 30 min $P K C \alpha A / 25 E$ group.

$\mathrm{AQP} 2$ as determined by AQP2 immunoprecipitation (Figure 4). These results were consistent with the AQP2 translocation observed by immunofluorescence detection and demonstrated that PKCa participated in DdAVP-mediated AQP2 translocation in $\mathrm{mIMCD}$ cells.

\section{Discussion}

PKCa exhibits different expression patterns in mouse and rat kidneys. For example, PKCa localizes in the proximal tubule of mice but not rats ${ }^{[3,4]}$. Our previous study demonstrated that PKCa contributes to urine concentration in the mouse kidney ${ }^{[5]}$. The use of cell lines that originate from mouse IMCD cells is reasonable because AQP2 is primarily expressed in collecting duct principal cells, and the effect of PKCa on urine concentrating occurs in the inner medulla. In the present study, AQP2 trafficking was examined in an MIMCD3 cell line that was derived from mouse IMCD and exhibited the properties of IMCD cells. However, immortalized MIMCD3 cells 
lack AQP2 expression ${ }^{[30]}$, and AQP2 is only briefly expressed in primary cultured IMCD cells ${ }^{[31]}$. Therefore, we generated AQP2-mIMCD3 stable cell lines for the following experiments. Our results demonstrated that AQP2 was located in intracellular vesicles in non-stimulated mIMCD3 cells. DdAVP treatment induced the translocation of AQP2 from the cytoplasm to the plasma membrane, and DdAVP washout reversed AQP2 localization from the plasma membrane to the cytoplasm (Figure 4). Therefore, AQP2-mIMCD3 cells provide an optimal cell model to investigate AQP2 trafficking.

AQP2 phosphorylation at serine 256 by PKA plays an important role in AQP2 trafficking ${ }^{[32,33]}$. However, the phosphorylation of AQP2 at serine 256 is not sufficient to maintain the presence of the water channel at the plasma membrane ${ }^{[34]}$, and the PKC-induced internalization of AQP2 in collecting duct cells does not depend on the phosphorylation state of $\mathrm{AQP2} 2^{[35,36]}$. Our study demonstrated that DdAVP increased AQP2 phosphorylation, and the inhibition of PKCa expression did not influence DdAVP-mediated AQP2 phosphorylation at serine 256, which is consistent with previous reports. These results strongly suggest that AQP2 phosphorylation at serine 256 is PKCa independent. However, the ability of PKCa to phosphorylate AQP2 at other phosphorylation sites, such as serine 231, remains unclear. Our results indicated that PKCa did not interact with AQP2 in vitro. Therefore, the ability of PKCa to mediate the trafficking of phosphorylated AQP2 requires further investigation.

The disruption of microtubules induces AQP2 translocation to the plasma membrane ${ }^{[13,23,29]}$, and PKCa is involved in cytoskeletal remodeling during cell motility, phagocytosis, neurite outgrowth and the regulation of cytoskeletonassociated proteins ${ }^{[37-40]}$. Therefore, the impact of PKCa on the cytoskeleton in AQP2-mIMCD3 cells was investigated. Microtubules are part of the cytoskeleton and consist of $\alpha, \beta$, and $\gamma$ tubulins ${ }^{[41]}$. a-Tubulin is the major cytoskeletal protein related to cell trafficking. The role of PKCa in the assembly of a-tubulin was determined. PKCa overexpression or inhibition produced a constitutively active form of PKCa or down-regulated expression of $\mathrm{PKCa}$, respectively, which resulted in the de- and re-polymerization of a-tubulin, respectively. Moreover, PKCa down-regulation prevented DdAVP-mediated a-tubulin depolymerization. These data indicated that $\mathrm{PKCa}$ is involved in the assembly of a-tubulin in AQP2-mIMCD3 cells, which is consistent with previous reports ${ }^{[9]}$.

The microtubule-dependent transport of AQP2 is predominantly responsible for AQP2 trafficking and localization inside of the cell after its internalization, but it is not responsible for the exocytic transport of the water channel ${ }^{[25]}$. In the present study, the overexpression of constitutively active PKCa produced a wide distribution of AQP2 throughout the cytoplasm, which is in contrast to its translocation to the plasma membrane. This result indicates that other factors/proteins rather than a-tubulin are required for AQP2 translocation to the plasma membrane ${ }^{[40]}$. Furthermore, the consistent inhibition of a-tubulin depolymerization by the down-regulation of PKCa expression produced a loss in DdAVP-mediated AQP2 translocation. This result confirms that a-tubulin is a key element for the proper localization of AQP2 in the cytoplasmic compartment.

In summary, the use of stably expressing AQP2-mIMCD3 cells demonstrated that $\mathrm{PKCa}$ did not functionally associate with AQP2. The down-regulation of PKCa expression altered the distribution of a-tubulin and inhibited DdAVP-mediated AQP2 trafficking. However, constitutively activated PKCa rescued or aggravated these changes. These data directly demonstrated that PKCa mediates AQP2 trafficking by influencing the assembly of a-tubulin and underscore the complexity of the molecular events of PKCa-mediated urine concentration.

\section{Acknowledgements}

This work was supported by the National Natural Science Foundation of China (№ 30871173).

\section{Author contribution}

Dr Li-jun YAO designed the research and wrote the paper; Dr Hong ZHAO performed biotinlyation of cell surface protein experiment; Xi YAO performed Western blot and immunocytochemistry experiment; Tao-xia WANG prepared plasmids and analysed data; Wen-min JIN performed cell culture experiment; Qian-qian JI contributed reagents preparation; Dr Xiao YANG wrote the paper; Dr Qiu-hong DUAN performed the research.

\section{References}

1 Mellor H, Parker PJ. The extended protein kinase C superfamily. Biochem J 1998; 332: 281-92.

2 Ostlund E, Mendez CF, Jacobsson G, Fryckstedt J, Meister B, Aperia A. Expression of protein kinase $C$ isoforms in renal tissue. Kidney Int 1995; 47: 766-73.

3 Pfaff IL, Wagner HJ, Vallon V. Immunolocalization of protein kinase C isoenzymes alpha, beta1 and betall in rat kidney. J Am Soc Nephrol 1999; 10: 1861-73.

4 Redling S, Pfaff IL, Leitges M, Vallon V. Immunolocalization of protein kinase $\mathrm{C}$ isoenzymes alpha, beta I, beta II, delta, and epsilon in mouse kidney. Am J Physiol Renal Physiol 2004; 287: F289-F298.

5 Yao L, Huang DY, Pfaff IL, Nie X, Leitges M, Vallon V. Evidence for a role of protein kinase $\mathrm{C}$-alpha in urine concentration. Am J Physiol Renal Physiol 2004; 287: F299-304.

6 Yao LJ, Leitges M, Vallon V. Mice lacking protein kinase $\mathrm{C}$ beta present modest increases in systolic blood pressure and $\mathrm{NH}_{4} \mathrm{Cl}$ induced metabolic acidosis. Kidney Blood Press Res 2006; 29: 36-42.

7 Kim JH, Lee-Kwon W, Park JB, Ryu SH, Yun CH, Donowitz M. $\mathrm{Ca}^{2+}$ dependent inhibition of $\mathrm{Na}^{+} / \mathrm{H}^{+}$exchanger 3 (NHE3) requires an NHE3-E3KARP-alpha-actinin-4 complex for oligomerization and endocytosis. J Biol Chem 2002; 277: 23714-24.

8 Qualmann B, Kessels MM, Kelly RB. Molecular links between endocytosis and the actin cytoskeleton. J Cell Biol 2000; 150: F111-F116.

9 Hryciw DH, Pollock CA, Poronnik P. PKC-alpha-mediated remodeling of the actin cytoskeleton is involved in constitutive albumin uptake by proximal tubule cells. Am J Physiol Renal Physiol 2005; 288: F1227-F1235.

10 Fushimi K, Uchida S, Hara Y, Hirata Y, Marumo F, Sasaki S. Cloning 
and expression of apical membrane water channel of rat kidney collecting tubule. Nature 1993; 361: 549-52.

11 Nielsen S, DiGiovanni SR, Christensen El, Knepper MA, Harris HW. Cellular and subcellular immunolocalization of vasopressin-regulated water channel in rat kidney. Proc Natl Acad Sci U S A 1993; 90: 11663-7.

12 Takata K, Matsuzaki T, Tajika Y. Aquaporins: water channel proteins of the cell membrane. Prog Histochem Cytochem 2004; 39: 1-83.

13 Tajika Y, Matsuzaki T, Suzuki T, Ablimit A, Aoki T, Hagiwara H, et al. Differential regulation of AQP2 trafficking in endosomes by microtubules and actin filaments. Histochem Cell Biol 2005; $124: 1$.

14 Rojek A, Fuchtbauer EM, Kwon TH, Frokiaer J, Nielsen S. Severe urinary concentrating defect in renal collecting duct-selective AQP2 conditional-knockout mice. Proc Natl Acad Sci U S A 2006; 103: 6037-42.

15 Takata K, Matsuzaki T, Tajika Y, Ablimit A, Hasegawa T. Localization and trafficking of aquaporin 2 in the kidney. Histochem Cell Biol 2008; 130: 197-209.

16 Nielsen S, Chou CL, Marples D, Christensen El, Kishore BK, Knepper MA. Vasopressin increases water permeability of kidney collecting duct by inducing translocation of aquaporin-CD water channels to plasma membrane. Proc Natl Acad Sci U S A 1995; 92: 1013-7.

17 Yamamoto T, Sasaki S, Fushimi K, Ishibashi K, Yaoita E, Kawasaki $\mathrm{K}$, et al. Vasopressin increases AQP-CD water channel in apical membrane of collecting duct cells in Brattleboro rats. Am J Physiol 1995; 268: C1546-C1551.

18 Boone M, Deen PM. Physiology and pathophysiology of the vasopressin-regulated renal water reabsorption. Pflugers Arch 2008; 456: 1005-24.

19 Hozawa S, Holtzman EJ, Ausiello DA. cAMP motifs regulating transcription in the aquaporin 2 gene. Am J Physiol 1996; 270 : C1695-C1702.

20 Matsumura Y, Uchida S, Rai T, Sasaki S, Marumo F. Transcriptional regulation of aquaporin-2 water channel gene by cAMP. J Am Soc Nephrol 1997; 8: 861-7.

21 Hasler U, Leroy V, Martin PY, Feraille E. Aquaporin-2 abundance in the renal collecting duct: new insights from cultured cell models. Am J Physiol Renal Physiol 2009; 297: F10-F18.

22 Li C, Wang W, Rivard CJ, Lanaspa MA, Summer S, Schrier RW. Molecular mechanisms of angiotensin II stimulation on aquaporin-2 expression and trafficking. Am J Physiol Renal Physiol 2011; 300: F1255-F1261.

23 Noda Y, Horikawa S, Kanda E, Yamashita M, Meng H, Eto K, et al. Reciprocal interaction with G-actin and tropomyosin is essential for aquaporin-2 trafficking. J Cell Biol 2008; 182: 587-601.

24 Takata K. Aquaporin-2 (AQP2): its intracellular compartment and trafficking. Cell Mol Biol 2006; 52: 34-9.

25 Vossenkamper A, Nedvetsky PI, Wiesner B, Furkert J, Rosenthal W, Klussmann E. Microtubules are needed for the perinuclear positioning of aquaporin-2 after its endocytic retrieval in renal principal cells. Am J Physiol Cell Physiol 2007; 293: C1129-C1138.

26 Baier-Bitterlich G, Uberall F, Bauer B, Fresser F, Wachter H, Grunicke $\mathrm{H}$, et al. Protein kinase $\mathrm{C}$-theta isoenzyme selective stimulation of the transcription factor complex AP-1 in T lymphocytes. Mol Cell Biol 1996; 16: 1842-50.
27 Goel M, Zuo CD, Schilling WP. Role of CAMP/PKA signaling cascade in vasopressin-induced trafficking of TRPC3 channels in principal cells of the collecting duct. Am J Physiol Renal Physiol 2010; 298 : F988-F996.

28 Hays RM, Condeelis J, Gao Y, Simon H, Ding G, Franki N. The effect of vasopressin on the cytoskeleton of the epithelial cell. Pediatr Nephrol 1993; 7: 672-9.

29 Nedvetsky PI, Tamma G, Beulshausen S, Valenti G, Rosenthal W, Klussmann E. Regulation of aquaporin-2 trafficking. Handb Exp Pharmacol 2009, 190: 133-57.

30 Umenishi F, Narikiyo T, Schrier RW. Effect on stability, degradation, expression, and targeting of aquaporin-2 water channel by hyperosmolality in renal epithelial cells. Biochem Biophys Res Commun 2005; 338: 1593-9.

31 Maric K, Oksche A, Rosenthal W. Aquaporin-2 expression in primary cultured rat inner medullary collecting duct cells. Am J Physiol 1998; 275: F796-801.

32 Katsura T, Gustafson CE, Ausiello DA, Brown D. Protein kinase A phosphorylation is involved in regulated exocytosis of aquaporin-2 in transfected LLC-PK1 cells. Am J Physiol 1997; 272: F817-F822.

33 Christensen BM, Zelenina M, Aperia A, Nielsen S. Localization and regulation of PKA-phosphorylated AQP2 in response to $\mathrm{V}(2)$-receptor agonist/antagonist treatment. Am J Physiol Renal Physiol 2000; 278: F29-F42.

34 Nejsum LN, Zelenina M, Aperia A, Frokiaer J, Nielsen S. Bidirectional regulation of AQP2 trafficking and recycling: involvement of AQP2S256 phosphorylation. Am J Physiol Renal Physiol 2005; 288 : F930-F938

35 Van Balkom BW, Savelkoul PJ, Markovich D, Hofman E, Nielsen S, van der Sluijs $\mathrm{P}$, et al. The role of putative phosphorylation sites in the targeting and shuttling of the aquaporin-2 water channel. J Biol Chem 2002; 277: 41473-9.

36 Kamsteeg EJ, Hendriks G, Boone M, Konings IB, Oorschot V, van der Sluijs $P$, et al. Short-chain ubiquitination mediates the regulated endocytosis of the aquaporin-2 water channel. Proc Natl Acad Sci U S A 2006; 103: 18344-9.

37 Holm A, Tejle K, Gunnarsson T, Magnusson KE, Descoteaux $A$, Rasmusson B. Role of protein kinase $C$ alpha for uptake of unopsonized prey and phagosomal maturation in macrophages. Biochem Biophys Res Commun 2003; 302: 653-8.

38 Beaudry H, Gendron L, Guimond MO, Payet MD, Gallo-Payet N. Involvement of protein kinase $\mathrm{C}$ alpha (PKC alpha) in the early action of angiotensin II type 2 (AT2) effects on neurite outgrowth in NG108-15 cells: AT2-receptor inhibits PKC alpha and p21ras activity. Endocrinology 2006; 147: 4263-72.

39 Belin RJ, Sumandea MP, Allen EJ, Schoenfelt K, Wang H, Solaro $\mathrm{RJ}$, et al. Augmented protein kinase C-alpha-induced myofilament protein phosphorylation contributes to myofilament dysfunction in experimental congestive heart failure. Circ Res 2007; 101: 195-204.

40 Abeyweera TP, Chen X, Rotenberg SA. Phosphorylation of alpha6tubulin by protein kinase Calpha activates motility of human breast cells. J Biol Chem 2009; 284: 17648-56.

41 Nogales E, Wolf SG, Downing KH. Structure of the alpha beta tubulin dimer by electron crystallography. Nature 1998; 391: 199-203. 